\title{
Social Referencing during Infancy and Early Childhood across Cultures
}

Christine Fawcett, Uppsala University, Uppsala, Sweden

Ulf Liszkowski, University of Hamburg, Hamburg, Germany

(c) 2015 Elsevier Ltd. All rights reserved.

\begin{abstract}
The article reviews the state of knowledge on infants' social referencing. The developmental course of social referencing throughout infancy is described, as well as current debates on the motivation behind social referencing. Variation in social referencing depending on the type or information, information source, and characteristics of the infant are described. Finally, cultural variation and the presence of social referencing in nonhumans are discussed.
\end{abstract}

\section{Classic Studies in Social Referencing}

Social referencing describes the tendency to gather information from other individuals to regulate one's own behavior in ambiguous situations. Social referencing was first studied in adults (e.g., Asch, 1952; Schachter and Singer, 1962; Sherif, 1958), and until the 1980s, it was an open question whether infants would also show this behavior. Yet it seemed likely that they would, given that the literature on attachment, showing that infants see their caregivers as a secure base for exploration (e.g., Ainsworth et al., 1979) suggests that they could also see caregivers as a source of information about the world (Feinman and Lewis, 1983).

An additional motivation for studying social referencing in infants was to show that emotional expressions themselves were more than by-products of an emotional experience, but actually used as signals to others that could alter behavior (Sorce et al., 1985). If infants change their behavior based solely on the emotional expressions of others, then those expressions must have referential meaning. The emotion is about something in the world and thus can provide useful information for the infant.

In one of the earliest social referencing studies with infants, Feinman and Lewis (1983) examined 10-month-old infants behavior toward a stranger when their mother gave positive or neutral signals about the person either directly to the infant or indirectly - when speaking to the stranger herself. They also measured infants' temperament and found that infants with an easy temperament who received direct positive information were more likely to approach the stranger. There were no other differences observed. However, it is possible that the situation was actually not ambiguous for most infants; thus the information from their caregiver did not lead them to adjust their behavior. That is, if infants know that a situation is safe or dangerous, they should not look for guidance from others.

The importance of uncertainty for social referencing was demonstrated by Sorce et al. (1985). Their study used a visual cliff with varying depths. That is, 12 -month-old infants could cross from a normal-looking floor to a glass side with the floor seemingly close or far below them. When the floor was far, infants were unsure about whether crossing was safe and thus looked to their mother for information. If their mother's response was positive (i.e., a facial expression of happiness or interest), the majority of infants crossed the visual cliff. In contrast, if the mother's expression showed fear or anger, very few infants crossed. Their study also showed the importance of an ambiguous situation for social referencing to occur. If the floor of the visual cliff did not appear to drop off at all, infants crossed even when their mother's expression was of fear. In fact, infants rarely looked to her in this situation, and even those who did continued to cross, failing to change their behavior.

In another classic study, Hornik and Gunnar (1988) described the social referencing behavior of 12 - and 18-month-old infants toward their caregiver in a more natural setting. This allowed them to examine the interplay of social referencing with other kinds of communication, such as affect sharing. Infants were presented with a rabbit in a cage and their behavior toward their mother was examined. The results showed that infants' referencing looks - those paired with a puzzled or wary facial expression - were as likely as affect-sharing looks when the rabbit was first introduced, and over time, referencing looks occurred much less often than sharing looks. This suggests that infants gather the information they need early on, but continue to want to share an experience with others over time. A second interesting finding from the study was that infants classified as initially wary or bold regarding the rabbit engaged in the same amount of referencing to the caregiver. This may show that social referencing can be used not only to seek valenced information (e.g., is it safe to approach?), but also instrumental information (e.g., what is this?) about an ambiguous situation.

This early work on social referencing in infancy served to establish several typical methodologies and to set the groundwork for future research that would more closely examine the process and development of social referencing.

\section{The Developmental Course of Social Referencing}

The first studies on social referencing focused on children around 1 year of age. How early do infants reference others for information when they are in ambiguous situations? A further question is whether referencing (i.e., seeking information) and behavioral regulation (i.e., acting on that information) develop at the same time. 


\section{Earliest Evidence of Social Referencing}

Using a cross-sectional design, Walden and Ogan (1988) explored the emergence of social referencing behaviors between 6 and 22 months of age. They found that at younger ages (6-9 months), infants were less discriminatory in their gaze to parents. When they looked at their parent, they did not always look to her face, suggesting that they may have only been checking that she was available, rather than looking for information. Younger infants also tended to look more to their parents when positive expressions were conveyed, whereas older infants (14-22 months) looked more to their parent's fearful expressions. Infants did not reliably regulate their behavior based on their parent's expression until between 10 and 13 months. At that age, infants were less likely to approach a toy after a fear reaction from the parent than a positive reaction. In addition, older infants were the only ones more likely to look to their parent before touching the toy. Interestingly, during a later free play trial after the parent was no longer giving emotional reactions, the older infants in their study (14-22 months) were actually more likely to touch the toy that had received a fear reaction, whereas the younger two groups were less likely to. In that situation, the toys were no longer moving or making noise and the experimenter invited infants to play with them. Thus, older infants may have inferred that the previously fearful toy was now safe. Together, this study suggests that social referencing behavior emerges around 1 year of age, but that it also continues to develop through the second year of life. Yet with a different experimental design, perhaps infants would be able to show social referencing at even earlier ages.

Until recently, the earliest evidence of behavioral regulation in response to other's emotional reactions was found at 8.5 months of age. Boccia and Campos (1989) found that infants were more wary of a stranger if their mother reacted with a stern rather than a cheerful greeting when the stranger entered the room. However, a recent study now shows that already by 5.5 months of age, infants are sensitive to emotionobject relations and regulate their own behavior based on those relations, but only for stimuli that had both facial and vocal emotional information (Vaillant-Molina and Bahrick, 2012). In a looking-time paradigm, infants demonstrated recognition of the specificity of another's emotional reactions by showing increased attention when the actor reversed her emotional reactions of happiness and fear to two different toys. Interestingly, they also preferred to touch the toy previously associated with a happy reaction. This suggests that they were also regulating their own behavior based on the actor's happy and fearful reactions, although they did not have to seek out the information as in typical social referencing paradigms. Another difference between this study and the standard ones is that it used habituation. Thus, the young infants were given as many repetitions of the emotional information as needed to fully process it. This extensive exposure could be one reason why infants were able to show social referencing ability at such a young age.

\section{What Changes over Development?}

Social referencing requires the ability to seek out information when needed, understand others' emotions, apply those emotions to objects or situations in the world, and to regulate behavior based on the information given. Infants' development in these areas can help give insight into when and how their social referencing develops and increases in sophistication.

\section{Information-Seeking}

Information-seeking may actually be one of the latest developing aspects of social referencing. Baldwin and Moses (1996) proposed that even though 12-month-old infants can make use of others' emotions to regulate their behavior, classic social referencing paradigms do not give clear evidence that infants actively seek out emotional information in an ambiguous situation. Instead, they may simply come across the information when they happen to look to an adult for other reasons, such as comfort-seeking or checking whether the caregiver is available. A likely prerequisite for information seeking is a concept of knowledge and ignorance. Infants must recognize that they lack information in an ambiguous situation and that another person may have useful information. It is an ongoing debate whether infants have stable concepts of knowledge and ignorance at 12 months of age (e.g., Liszkowski et al., 2008; Surian et al., 2007). Walden and Ogan (1988) found that only infants over 14 months of age are likely to check with their parent before approaching an ambiguous toy, even though younger infants' approach behavior is regulated by the caregiver's reaction.

A few studies have examined information-seeking, though not necessarily in a social-referencing context. Vaish et al. (2011) used an object-labeling paradigm and showed that 13- and 18-month-old infants were more likely to look to the person who labeled an object when it was necessary to disambiguate her label. That is, when two objects were available, infants checked her gaze to determine which one she was attending to, whereas when only one object was available, they were less likely to do so. Infants may also seek information when pointing at things (Baldwin and Moses, 1996; Liszkowski, 2005). Begus and Southgate (2012) looked at pointing behavior to determine whether infants seek information appropriately from others. They found that 16-month-olds were more likely to point to novel objects when interacting with a knowledgeable experimenter than an ignorant one, suggesting that they knew the knowledgeable person would be able to provide them with more information. While neither of these studies directly tested informationseeking in a social referencing paradigm, they do suggest that at least by early in the second year of life, infants can seek out information from others in a sophisticated way.

\section{Emotion Recognition}

Young infants are not yet able to approach and avoid situations in the same way as older infants can; however, sensitive measures of infants' attention can indicate some understanding of emotional reactions to objects. Hoehl et al. (2008) looked at whether very young infants' brain responses indicated that they were already sensitive to when another person reacted negatively to an object. They showed that infants' neural responses to objects differed if those objects had previously been shown in the context of a person gazing at the object with a negative emotional expression. This suggests that already at 3 months of age, infants can associate others' negative emotional reactions with objects and they 
subsequently pay more attention to those objects, even when the emotional information is no longer visible.

The Hoehl et al. (2008) study only compared negative emotions to neutral expressions. Yet we also know that between 3 and 4 months of age, infants begin to discriminate between multimodal emotional expressions of happiness, anger, and sadness, as indicated by preferentially gazing at novel facial expressions in a habituation paradigm (Flom and Bahrick, 2007). By 7 months of age, infants also show increased attention for fearful facial expressions in that they have difficulty disengaging from a face with a fear expression (Peltola et al., 2008, 2009).

Studies on emotion differentiation cannot speak to whether infants recognize the meaning behind the expression they observe or whether infants' own emotional experience is affected in any way. Yet they show that infants have the first abilities necessary to begin learning more about the relevance of emotions in their world.

\section{Referential Understanding}

In addition to understanding what emotion another is expressing, social referencing requires that infants recognize the referent of the emotional reaction. Moses et al. (2001) showed that both 18- and 12-month-old infants appreciated the relevance of an emotional message when regulating their behavior toward an ambiguous toy. That is, an emotional reaction was delivered either by someone attending or not attending to the ambiguous toy. Infants were selective such that they differed in their approach to the toy based on positive and negative emotional reactions only for the toy to which the reaction was directed. For example, if the infant was examining one toy while the experimenter emoted toward a different toy, infants appropriately paired the reaction with the toy in the experimenter's view.

Repacholi (1998) also showed that 14- and 18-month-old infants have referential understanding of others' emotional reactions. Infants observed an adult gaze into each of two boxes and react positively to one and negatively to the other. Later, infants were more likely to explore the contents of the box that had been reacted to positively, even though they were equally likely to touch both boxes themselves. This suggests that infants accurately applied the emotional reactions of the adult to the contents of the box - even though they were not visible to the infant at the time - and not the box itself. Thus, early in the second year of life, infants seem to be able to accurately apply emotional messages to objects and situations.

\section{Locomotion}

A proposed factor influencing changes in social referencing around 8-9 months of age is the onset of crawling. Since crawling allows infants to be more independent from their caregivers, the need for social referencing and the distal communication it provides likely increases sharply once infants begin to crawl. Infants who have begun to crawl pay more attention to distant objects and check back with their caregiver more often than infants of the same age who are not yet crawling (Campos et al., 1992). Crawling infants also receive more prohibitions from their caregivers, particularly when the infant is far from the caregiver (Zumbahlen and
Crawley, 1996). Together these findings from interview studies suggest that crawling may be a catalyst for social referencing behavior. In addition, infants with locomotor experience are more able to follow the referential gestures (gaze and pointing) of others, another important skill for social referencing (Campos et al., 2000).

There is also some evidence that the transition from crawling to walking could be influential in the development of social referencing. One study suggested that when infants begin to walk, they actually begin to socially reference less and engage in more social bidding than younger, crawling infants (Clearfield et al., 2008). However, it is possible that the novel toys used in that particular study may not have been sufficiently ambiguous to elicit social referencing behavior in the older, walking infants.

\section{Can Mood Explain Social Referencing?}

Given that evidence suggests that infants already understand the referential nature of emotion when they engage in social referencing, it is unlikely that mood can account for behavioral differences seen in social referencing situations. In addition, many studies have specifically ruled out mood as a confounding variable. For example, Hornik et al. (1987) found that caregivers' emotional reactions affected infants' behavior toward the particular toy that was the target of their reaction, but not to infants play behavior in general. Similarly, Feinman and Lewis (1983) accounted for mood by comparing infants' behavior toward a stranger (who had been reacted to either positively or negatively) to behavior toward their mother. Even when behavior toward their mother was subtracted, there was a difference in approach to the stranger based on the mother's expression, showing that mood and general willingness to approach another did not explain their findings. Walden and Ogan (1988) examined infants' own emotional expression during a social referencing task and again found no evidence that mood could be a confounding factor.

Finally, Stenberg and Hagekull (1997) directly tested the possibility of mood modification by having caregivers deliver either specific (directed to a particular object) or general positive or negative emotional messages. They found that while infants' behavior toward the toy was affected by the specific emotional messages, with more play following the positive than negative reaction, infants' overall activity was affected by the general emotional message. The findings suggest that when emotional information is specifically directed to an object, infants' own mood is not a factor in their behavior. In contrast, when general positive or negative emotional information is presented, infants do not have a clear target for that expression and react with overall differences in their exploratory behavior.

\section{Whom Do Infants Prefer to Reference?}

Do infants preferentially reference certain people over others? Research in this area has focused on infants' tendency to reference their caregivers compared to a familiar experimenter. The debate is rooted in the question of whether infants' looks 
in ambiguous situations are information-seeking behaviors related to social referencing, or rather comfort-seeking behaviors related to attachment. In one such study, Zarbatany and Lamb (1985) presented infants with an unusual toy and either their mother or an experimenter was in the room posing fear or smiling expressions. Infants were found to reference both their mother and the experimenter, but their behavior toward the toy was only influenced by the reactions of the mother. When the experimenter was posing either expression, infants were unlikely to approach the toy. It was only when the mother showed a positive expression that they did so. A later study using a similar paradigm, but with both adults remaining in the room for the duration of the study, gave contrasting results. Infants both referenced the experimenter and regulated their behavior based on her reactions, with fewer and slower approaches to the toy following fear than smiling expressions (Klinnert et al., 1986). Thus, it is possible that infants in the Zarbatany and Lamb study were too uncomfortable in the room without their caregiver present that they were not able to approach the toy, even when the experimenter's expression was positive.

More recent studies have continued to find that infants tend to engage in more social referencing toward an experimenter than their caregiver. They do so not only when their parent is inattentive (Stenberg, 2003), but also when both parent and experimenter are providing information. Specifically, Walden and Kim (2005) presented 18- and 24-month-old infants with ambiguous toys and both caregiver and experimenter provided emotional reactions about the toy when infants looked toward them. They found that infants at both ages looked more toward the experimenter than their mother and that this difference was even greater for the 24-month-olds. Importantly, in a baseline looking measure, infants looked equally to their mother and the experimenter, ruling out overall interest in the experimenter as an explanation for the difference in referencing.

Stenberg (2009) found that infants' preference for referencing experimenters holds even when the caregiver is the one introducing the ambiguous toy and providing the emotional reaction about it. That study also looked specifically at 12-month-old infants' behavioral regulation following positive emotional reactions delivered by either the caregiver or experimenter. The findings showed that infants played longer with the toy following positive messages from the experimenter than the mother. However, since the study only included positive emotional reactions, it's not possible to say whether the pattern of findings would hold if the experimenter and caregiver were giving negative, rather than positive, messages.

Together, the findings suggest that infants may be relying on an inference of expertise when selecting whom to reference. That is, they look to the experimenter and trust her information more because they expect that the experimenter will know more about the ambiguous object or situation.

\section{The Effect of Information Type on Social Referencing}

What kind of information do infants use when socially referencing others? The modality of information - visual versus vocal, as well as the valence of the information being delivered could have differential effects on later behavioral regulation.

\section{Modality}

Multimodal emotional expressions - displayed with both facial and vocal information - are those earliest discriminated by infants. Flom and Bahrick (2007) showed that by 4 months of age, infants could discriminate multimodal expressions of happiness, sadness, and anger, and that they could only do so when temporal synchrony between the face and voice were intact. By 5 months, infants could discriminate the expressions with only vocal information and by 7 months they could do so with only visual information available. The same developmental trajectory for emotional expressions seems to be seen in social referencing. Twelve-month-old infants' crossing of a visual cliff was affected by multimodal and vocal emotional expressions, but not expressions of the face alone (Vaish and Striano, 2004).

In a more naturalistic study of the modality for social referencing messages, caregivers were asked to improvise their own emotional messages. Results showed that they tended to produce more gestures with messages of fear and more vocalizations with positive messages (Kim et al., 2010). Further, these differences were also what infants responded best to. They modified their behavior more based on multimodal and visual information in the case of fear and based on the amount of vocal information in the case of a positive message.

Even when the modality for an emotional message is video, infants regulate their behavior based on it. Specifically, Mumme and Fernald (2003) found that 12-month-old infants, but not 10-month-old infants, were less likely to touch a toy when they had seen someone on video react negatively toward it, compared to a toy that was reacted to positively or neutrally.

\section{Valence}

Are both positive and negative types of information valuable during social referencing? Vaish et al. (2008) propose that there is a negativity bias in social referencing, such that infants' behavior is more affected by negative than positive emotional reactions. When a neutral baseline is used as a comparison, most social referencing studies find little difference between infants' behavioral responses to positive versus neutral reactions from others, yet a significant difference between neutral and negative remains (e.g., Hornik et al., 1987; Mumme et al., 1996). This is important because it shows that social referencing may function primarily in one direction - encouraging infants to avoid objects and situations, but not to explore them.

Looking at valence from the caregiver's perspective, mothers naturally gave more intense positive reactions than negative ones when asked to improvise their own messages to infants (Kim et al., 2010). It could be that caregivers already know that a positive emotional message needs to be higher in intensity to influence their infant's behavior compared to a negative message. 


\section{Individual Differences in Social Referencing}

\section{Temperament}

Findings on the relation between social referencing behavior and temperament have been somewhat mixed. Temperament refers to stable characteristics including emotional valence, fearfulness, inhibition, and ease of being comforted. It could be that infants who are more temperamentally fearful or less easily comforted would be more likely to reference others when encountering ambiguous situations or more likely to act on the information they receive during social referencing.

In one of the earliest studies on infant social referencing, Feinman and Lewis (1983) found that infants with easy, but not difficult temperaments increased proximity and offered more toys to a stranger following positive messages about the stranger from their mother. In contrast, Bradshaw et al. (1987) later found no relation between behavioral regulation during social referencing and positive/negative affect temperament, which corresponds to the easy/difficult dimension used by Feinman and Lewis. They did, however, find that infants with a more positive temperament made more social referencing looks to their caregiver.

Blackford and Walden (1998) investigated the role of temperamental fear on social referencing behavior. They found that low-fear 11- to 13-month-old infants were more likely to regulate their behavior based on positive or fearful messages from their caregiver regarding a novel toy than fearful infants who tended to avoid the toy altogether. Temperament did not have an effect on how often infants looked to their caregivers. Thus, the seeking of information remained the same, but the use of information differed for infants with different temperaments.

It is possible that the effect of temperament, at least fearful temperament, comes down to how ambiguous the experimental situation is for the infant. If the infant is temperamentally fearful, then he or she is already predisposed to avoid to the toy, despite a positive message from an adult. This is an important confound to keep in mind, since ambiguity is important for an accurate measure of social referencing.

\section{Attachment Style}

Social referencing and attachment are in some sense very closely intertwined. There is still some debate as to whether social referencing looks are actually comfort-seeking behavior, related to attachment, rather than information-seeking behavior (see Section Whom Do Infants Prefer to Reference? earlier in the article). However, even if social referencing is based on information-seeking, infants could differ in how likely they are to seek information from their caregiver depending on the quality of their attachment relationship. For example, in a study relating attachment security to referencing behavior, insecure-avoidant infants referenced their parent the least and insecure-resistant infants referenced the most, with securely attached infants in between (Dickstein et al., 1984). This is in line with the definitions of insecure attachment styles, in which avoidant infants tend to ignore their attachment figures, even in ambiguous situations, and resistant infants continue to seek comfort, but fail to actually be comforted by the attachment figure (Ainsworth et al., 1979).

\section{Atypical Development}

Children with autism spectrum disorder (ASD) tend to have significant deficits in social cognition, including social orienting, joint attention, and attention to others' distress (Dawson et al., 2004). Thus, it seems likely children with ASD would show impairments in social referencing. Sigman et al. (1992) found that 3- to 4-year-old children with ASD were less likely to respond to adults' emotional reactions than control participants, yet those who did respond regulated their behavior toward the toy accordingly. Their study could not examine referential looking, however, since the adult expressed an emotional reaction regardless of whether the child looked at her.

Since ASD is not typically diagnosed until 3 years of age, one recent study examined the social referencing abilities of infant siblings of children with ASD, who are themselves at high risk for developing the disorder (Cornew et al., 2012). For referential looking, infants who were later diagnosed as having autism were slower, and overall less likely, to spontaneously look to an adult when an ambigious toy was present, compared to typically developing infants. Interestingly, behavioral regulation based on others' emotional signals was found to be impaired in infant siblings of autistic children, regardless of whether they were later diagnosed with autism or not. Only typically developing control participants showed appropriate behavioral regulation in the study. The authors suggest that there may be a more subtle impairment present in the high-risk infants who do not develop ASD that accounts for their decreased social referencing.

\section{Cultural Differences in Social Referencing}

To our knowledge, no studies have specifically examined crosscultural differences in social referencing. Even though cultures differ in their forms of communication, parenting practices, and social norms, some aspects of communication and emotion seem to be largely universal. For example, the expression and recognition of emotions (see Elfenbein and Ambady, 2002, for a review) and infants' reference to objects by pointing (Liszkowski et al., 2012) seem to be universal. In addition, an extensive study of early social cognitive skills in three cultures (Canada, India, and Peru) showed that gaze following and joint attention were not significantly different, despite widely varying parenting practices and social norms (Callaghan et al., 2011). Cultural differences have been reported in the frequency of mutual gaze in dyadic face-to-face interactions of parents and their young infants, with Nso parents engaging in less direct eye contact than German parents (Kärtner et al., 2010; Keller et al., 2011). However, it is unknown whether there is a relation between early mutual gaze and the later emergence of social referencing.

With little variation in the basic skills of emotion recognition, gaze following, and joint attention, it seems likely that social referencing would also be present cross-culturally. The primary difference in social referencing across cultures may rather be in the content of what children learn, rather than the use of referencing itself. That is, the mechanism of social 
referencing may be universal, but the information gained through social referencing regarding what should be approached or avoided could depend on what culture the infant is a part of. A second possible difference could be in the individuals targeted during social referencing. For example, if children in some cultures spend more time with siblings than their parents (e.g., Harkness and Super, 1995), older children could be a more common source for social referencing information.

\section{Social Referencing in Nonhumans}

Social referencing does not seem to be an ability that is specific to human infants. Itakura (1995) observed motherinfant pairs of chimpanzees and found that infants were more likely to seek contact with their mother when novel objects were present, suggesting that they could be engaging in social referencing. In a more controlled experiment, similar to those used with infants, Russell et al. (1997) demonstrated that young chimpanzees made referential looks to a familiar human caregiver and modified their behavior toward novel toys based on the caregiver's reactions of happiness or fear.

Macaque monkeys were also found to develop social referencing behavior. Older (5- to 12-months), but not younger infant macaques made more looks to their mothers in the presence of a rubber snake than in a control condition with no snake present (Roberts et al., 2008). Yet when clear referential looks - looking to the mother directly before or after looking to the snake - were measured, only $13 \%$ of the macaques were found to engage in looking from the snake to their mother. Also, since the mother's emotional reaction could not be manipulated, it is not known whether the infant macaques also regulate their behavior based on her reaction. Yet this study does give some evidence that macaques look to their mothers when an ambiguous object is present, whether for information or comfort.

Domestic dogs were found to socially reference their owners regarding an ambiguous object (Merola et al., 2012a). All dogs in their study looked to their owner after looking at the ambiguous object, demonstrating robust referential looking. There was also some evidence for behavioral regulation in that dogs that received a negative message from their owners were more likely to stop moving around the room than those who received a positive message. Additionally, dogs were found to approach the object more if their owner also approached it than if their owner avoided it. In a later study, Merola et al. (2012b) found that dogs looked not only to their owner, but also to a stranger for social referencing. There was also clearer evidence for behavioral regulation in dogs, at least when the owner was providing the emotional message. Dogs that received a positive message were more likely to approach the object than those who received a negative message.

Together these studies show that several nonhuman species can engage in some aspects of social referencing. This is not altogether surprising given that primates and domestic dogs also show abilities related to social referencing, such as gaze following (e.g., Kaminski et al., 2009; Call et al., 1998). It remains to be seen whether other nonhuman species with sophisticated social cognitive skills, such as dolphins (Pack and Herman, 2006), also engage in social referencing.

\section{Conclusions and Future Directions}

Social referencing is an important part of how infants and young children learn about their world from social figures. From around the end of the first year, infants begin showing social referencing behavior - looking to others for guidance in ambiguous situations and regulating their behavior based on others' reactions. While social referencing is likely to be a universal skill, there are still individual, and possibly cultural variations. More work looking at these variations can help to give insight into the processes involved in developing social referencing and how it can be encouraged in children with autism, for example, who have impairments in this ability.

See also: Attachment During Infancy and Early Childhood:
Cultural Variations, Development of; Autism Spectrum
Disorders; Early Emotional Development and Cultural
Variability; Emotion in Cognition; Emotion: History of the
Concept; Emotional Development, Effects of Parenting and
Family Structure on; Emotional Regulation; Infancy and
Childhood: Emotional Development; Infancy and Human
Development; Neonatal Temperament: From Phenomenology
to Mechanism; Temperament and Human Development;
Temperament.

\section{Bibliography}

Ainsworth, M.D.S., Blehar, M.C., Waters, E., Wall, S., 1979. Patterns of Attachment: a Psychological Study of the Strange Situation. Lawrence Erlbaum, Hillsdale, NJ. Asch, R., 1952. Social Psychology. Prentice-Hall, Englewood Cliffs, NJ.

Baldwin, D.A., Moses, L.J., 1996. The ontogeny of social information gathering. Child Development 67 (5), 1915-1939.

Begus, K., Southgate, V., 2012. Infant pointing serves an interrogative function. Developmental Science 15 (5), 611-617.

Blackford, J.U., Walden, T.A., 1998. Individual differences in social referencing. Infant Behavior and Development 21 (1), 89-102.

Boccia, M., Campos, J.J., 1989. Maternal emotional signals, social referencing, and infants' reactions to strangers. New Directions for Child Development 44, 25-49.

Bradshaw, D.L., Goldsmith, H.H., Campos, J.J., 1987. Attachment, temperament, and social referencing: Interrelationships among three domains of infant affective behavior. Infant Behavior and Development 10 (2), 223-231. http://dx.doi.org/ 10.1016/0163-6383(87)90036-1.

Call, J., Hare, B., Tomasello, M., 1998. Chimpanzee gaze following in an object-choice task. Animal Cognition 1 (2), 89-99. http://dx.doi.org/10.1007/s100710050013.

Callaghan, T., Moll, H., Rakoczy, H., Warneken, F., Liszkowski, U., Behne, T., Tomasello, M., 2011. Early social cognition in three cultural contexts. Monographs of the Society for Research in Child Development 2, 76.

Campos, J.J., Anderson, D.I., Barbu-Roth, M.A., Hubbard, E.M., Hertenstein, M.J., Witherington, D., 2000. Travel broadens the mind. Infancy 1 (2), 149-219.

Campos, J.J., Kermoian, R., Zumbahlen, M.R., 1992. Socioemotional transformations in the family system following infant crawling onset. New Directions for Child and Adolescent Development 1992 (55), 25-40.

Clearfield, M.W., Osborne, C.N., Mullen, M., 2008. Learning by looking: infants' social looking behavior across the transition from crawling to walking. Journal of Experimental Child Psychology 100 (4), 297-307. http://dx.doi.org/10.1016/ j.jecp.2008.03.005

Cornew, L., Dobkins, K.R., Akshoomoff, N., McCleery, J.P., Carver, L.J., 2012. Atypical social referencing in infant siblings of children with autism Spectrum 
disorders. Journal of Autism and Developmental Disorders. http://dx.doi.org/ 10.1007/s10803-012-1518-8.

Dawson, G., Toth, K., Abbott, R., Osterling, J., Munson, J., Estes, A., Liaw, J., 2004 Early social attention impairments in autism: social orienting, joint attention, and attention to distress. Developmental Psychology 40 (2), 271.

Dickstein, S., Thompson, R.A., Estes, D., Malkin, C., Lamb, M.E., 1984. Socia referencing and the security of attachment. Infant Behavior and Development 7 (4), 507-516.

Elfenbein, H.A., Ambady, N., 2002. On the universality and cultural specificity of emotion recognition: a meta-analysis. Psychological Bulletin 128 (2), 203.

Feinman, S., Lewis, M., 1983. Social referencing at ten months: a second-order effect on infants' responses to strangers. Child Development 54 (4), 878-887. Retrieved from: http://www.ncbi.nlm. nih. gov/pubmed/6617309.

Flom, R., Bahrick, L.E., 2007. The development of infant discrimination of affect in multimodal and unimodal stimulation: the role of intersensory redundancy. Developmental Psychology 43 (1), 238-252. http://dx.doi.org/10.1037/00121649.43.1.238.

Harkness, S., Super, C.M., 1995. Culture and parenting. In: Bornstein, M.H. (Ed.) Handbook of Parenting: Biology and Ecology of Parenting, vol. 2. Lawrence Erlbaum, Mahwah, NJ, pp. 211-234.

Hoehl, S., Wiese, L., Striano, T., 2008. Young infants' neural processing of objects is affected by eye gaze direction and emotional expression. PloS One 3 (6), e2389. http://dx.doi.org/10.1371/journal. pone.0002389.

Hornik, R., Gunnar, M.R., 1988. A descriptive analysis of infant social referencing. Child Development 59 (3), 626-634. Retrieved from: http://www.ncbi.nlm.nih.gov/ pubmed/3383671.

Hornik, R., Risenhoover, N., Gunnar, M., 1987. The effects of maternal positive, neutral, and negative affective communications on infant responses to new toys. Child Development, 937-944.

Itakura, S., 1995. An exploratory study of social referencing in chimpanzees. Folia Primatologica 64 (1-2), 44-48.

Kaminski, J., Brauer, J., Call, J., Tomasello, M., 2009. Domestic dogs are sensitive to a human's perspective. Behaviour 146 (7), 979-998.

Kärtner, J., Keller, H., Yovsi, R.D., 2010. Mother-infant interaction during the first 3 months: the emergence of culture-specific contingency patterns. Child Development 81 (2), 540-554.

Keller, H., Borke, J., Lamm, B., Lohaus, A., Yovsi, R.D., 2011. Developing patterns of parenting in two cultural communities. International Journal of Behavioral Development 35 (3), 233-245.

Kim, G., Walden, T.A., Knieps, L.J., 2010. Impact and characteristics of positive and fearful emotional messages during infant social referencing. Infant Behavior \& Development 33 (2), 189-195. http://dx.doi.org/10.1016/j.infbeh.2009.12.009.

Klinnert, M.D., Emde, R.N., Butterfield, P., Campos, J.J., 1986. Social referencing: the infant's use of emotional signals from a friendly adult with mother present. Developmental Psychology 22 (4), 427-432. http://dx.doi.org/10.1037//00121649.22.4.427.

Liszkowski, U., 2005. Human twelve-month-olds point cooperatively to share interest with and helpfully provide information for a communicative partner. Gesture 5 (12), $1-2$.

Liszkowski, U., Carpenter, M., Tomasello, M., 2008. Twelve-month-olds communicate helpfully and appropriately for knowledgeable and ignorant partners. Cognition 108 (3), 732-739.

Liszkowski, U., Brown, P., Callaghan, T., Takada, A., de Vos, C., 2012. A prelinguistic gestural universal of human communication. Cognitive Science.

Merola, I., Prato-Previde, E., Marshall-Pescini, S., 2012a. Social referencing in dogowner dyads? Animal Cognition 15 (2), 175-185.

Merola, I., Prato-Previde, E., Marshall-Pescini, S., 2012b. Dogs' social referencing towards owners and strangers. PloS One 7 (10), e47653.

Moses, L.J., Baldwin, D.A., Rosicky, J.G., Tidball, G., 2001. Evidence for referentia understanding in the emotions domain at twelve and eighteen months. Child Development 72 (3), 718-735.

Mumme, D.L., Fernald, A., 2003. The infant as onlooker: learning from emotional reactions observed in a television scenario. Child Development 74 (1), 221-237.
Mumme, D.L., Fernald, A., Herrera, C., 1996. Infants' responses to facial and vocal emotional signals in a social referencing paradigm. Child Development 67 (6) 3219-3237. Retrieved from: http://www.ncbi.nlm.nih.gov/pubmed/9071778.

Pack, A.A., Herman, L.M., 2006. Dolphin social cognition and joint attention: our current understanding. Aquatic Mammals 32 (4), 443-460. http://dx.doi.org/ 10.1578/AM.32.4.2006.443.

Peltola, M.J., Leppänen, J.M., Palokangas, T., Hietanen, J.K., 2008. Fearful faces modulate looking duration and attention disengagement in 7-month-old infants. Developmental Science 11 (1), 60-68. http://dx.doi.org/10.1111/j.14677687.2007.00659.x.

Peltola, M.J., Leppänen, J.M., Mäki, S., Hietanen, J.K., 2009. Emergence of enhanced attention to fearful faces between 5 and 7 months of age. Social Cognitive and Affective Neuroscience 4 (2), 134-142.

Repacholi, B.M., 1998. Infants' use of attentional cues to identify the referent of another person's emotional expression. Developmental Psychology 34 (5), 1017.

Roberts, S.G.B., McComb, K., Ruffman, T., 2008. An experimental investigation of referential looking in free-ranging Barbary macaques (Macaca sylvanus). Journal of Comparative Psychology (Washington, D.C.: 1983) 122 (1), 94-99. http:// dx.doi.org/10.1037/0735-7036.122.1.94

Russell, C.L., Bard, K.A., Adamson, L.B., 1997. Social referencing by young chimpanzees (Pan troglodytes). Retrieved from: Journal of Comparative Psychology (Washington, D.C.: 1983) 111 (2), 185-193 http://www.ncbi.nlm.nih.gov/ pubmed/9170283.

Schachter, S., Singer, J., 1962. Cognitive, social, and physiological determinants of emotional state. Psychological Review 69 (5), 379.

Sherif, M., 1958. Group influences upon the formation of norms and attitudes. Readings in Social Psychology 3, 219-232.

Sigman, M.D., Kasari, C., Kwon, J.H., Yirmiya, N., 1992. Responses to the negative emotions of others by autistic, mentally retarded, and normal children. Child Development 63 (4), 796-807.

Sorce, J.F., Emde, R.N., Campos, J.J., Klinnert, M.D., 1985. Maternal emotional signaling: Its effect on the visual cliff behavior of 1 -year-olds. Developmenta Psychology 21 (1), 195-200. http://dx.doi.org/10.1037//0012-1649.21.1.195.

Stenberg, G., 2003. Effects of maternal inattentiveness on infant social referencing. Infant and Child Development 12, 399-419.

Stenberg, G., 2009. Selectivity in infant social referencing. Infancy 14 (4), 457-473. http://dx.doi.org/10.1080/15250000902994115.

Stenberg, G., Hagekull, B., 1997. Social referencing and mood modification in 1-yearolds. Infant Behavior and Development 20 (2), 209-217.

Surian, L., Caldi, S., Sperber, D., 2007. Attribution of beliefs by 13-month-old infants. Psychological Science 18 (7), 580-586.

Vaillant-Molina, M., Bahrick, L.E., 2012. The role of intersensory redundancy in the emergence of social referencing in $5 \frac{1}{2}$-month-old infants. Developmental Psychology 48 (1), 1-9. http://dx.doi.org/10.1037/a0025263.

Vaish, A., Striano, T., 2004. Is visual reference necessary? Contributions of facia versus vocal cues in 12-month-olds ' social referencing behavior Amrisha Vaish and Tricia Striano. Developmental Science 7 (3), 261-269.

Vaish, A., Grossmann, T., Woodward, A., 2008. Not all emotions are created equal: the negativity bias in social-emotional development. Psychological Bulletin 134 (3) 383-403. http://dx.doi.org/10.1037/0033-2909.134.3.383.

Vaish, A., Demir, Ö.E., Baldwin, D., 2011. Thirteen-and 18-month-old infants recognize when they need referential information. Social Development 20 (3), 431-449.

Walden, T., Kim, G., 2005. Infants' social looking toward mothers and strangers. International Journal of Behavioral Development 29 (5), 356-360. http:// dx.doi.org/10.1080/01650250500166824.

Walden, T.A., Ogan, T.A., 1988. The development of social referencing. Child Development 59 (5), 1230-1240.

Zarbatany, L., Lamb, M.E., 1985. Social referencing as a function of information source: mothers versus strangers. Infant Behavior and Development 8 (1) 25-33.

Zumbahlen, M., Crawley, A., 1996. Infants' early referential behavior in prohibition contexts: the emergence of social referencing. Infant Behavior and Development 19,244 